\title{
Identification of novel rapamycin derivatives as low-level impurities in active pharmaceutical ingredients
}

\author{
Stephan G Zech, Michael Carr, Qurish K Mohemmad, Narayana I Narasimhan, Christopher Murray, \\ Leonard W Rozamus and David C Dalgarno
}

We describe the identification of novel rapamycin derivatives present as low-level impurities in active pharmaceutical ingredients using an integrated, multidisciplinary approach. Rapamycin, a fermentation-derived natural product is itself used clinically and provides the starting material for several rapamycin analog drugs, typically used in oncology. LC-MS proved a sensitive means to analyze impurity profiles in batches of rapamycin. MS fragmentation was used to gain structural insight into these impurities, usually fermentation by-products, structurally very similar to rapamycin. In cases where MS fragmentation was unable to provide unambiguous structural identification, the impurities were isolated and purified using orthogonal HPLC methods. Using the higher mass sensitivity of small-volume NMR microprobes, submilligram amounts of isolated impurities were sufficient for further characterization by multidimensional NMR spectroscopy. Full assignment of the ${ }^{1} \mathrm{H}$ and ${ }^{13} \mathrm{C}$ NMR signals revealed the structure of these impurities at an atomic level. This systematic workflow enabled the identification of several novel rapamycin congeners from active pharmaceutical ingredient without the need for large-scale isolation of impurities. For illustration, two novel rapamycin derivatives are described in this study: 12-ethyl-rapamycin and 33-ethyl-rapamycin, which exemplify previously unreported modifications on the carbon skeleton of the rapamycin macrocycle. The methodologies described here can be of wide use for identification of closely related structures found; for example as fermentation by-products, metabolites or degradants of natural product-based drugs.

The Journal of Antibiotics (2011) 64, 649-654; doi:10.1038/ja.2011.61; published online 27 July 2011

Keywords: chromatography; impurity isolation; mass spectrometry; natural products; NMR spectroscopy; pharmaceutical formulations

\section{INTRODUCTION}

Rapamycin (sirolimus) ${ }^{1}$ is a natural product isolated from Streptomyces rapamycinicus ${ }^{2}$ (formerly Streptomyces hygroscopicus) with antibiotic and antifungal activities, which itself has clinical applications; for example, as an immuno-supressant drug $^{3,4}$ and as the active agent in drug-eluting stents. ${ }^{5-7}$ Rapamycin and many of its derivatives function by inhibiting the kinase mammalian target of rapamycin, an important regulator of cell proliferation..$^{8-11}$ Furthermore, two semisynthetic rapamycin analogs, temsirolimus and everolimus, have been approved as mammalian target of rapamycin inhibitors for cancer treatment, while a third, ridaforolimus, has entered late-stage clinical trials; for example, for sarcoma and other tumors. ${ }^{10,12}$ All these analogs are modified at the C43 hydroxyl group of the cyclohexyl moiety of rapamycin and consequently use rapamycin, a fermentation-derived natural product, as a starting material in their chemical synthesis. ${ }^{13}$

As part of the new drug regulatory approval process, impurities in the active pharmaceutical ingredient (API) must be structurally characterized, ${ }^{14,15}$ if they exceed the identification threshold of $0.10 \%$. Many publications have described the identification of impurities in pharmaceutical agents using various technologies including LC-MS/MS, accurate mass determination and LC-NMR. ${ }^{16-19}$ However, in the case of natural product-based APIs, a number of factors complicate the exhaustive characterization of lowlevel impurities, leading to a formidable analytical challenge. First, the biosynthetic pathways in the fermentation process are expected to generate a variety of closely related structural analog impurities, many of which will be present in low levels (for example, $<0.2 \%$ by weight) in the compound of interest. The impurities are then difficult to resolve chromatographically, and hence are challenging to isolate for characterization. Second, the limited stability of some fermentationbased natural products under certain temperatures, solvents and $\mathrm{pH}$ limits may complicate the isolation of larger amounts of impurities free of degradation by-products, and may compromise the use of some less-sensitive analytical techniques. Finally, reversible structural isomerizations; for example, tautomerization, and the presence 
of rotamers can complicate both impurity isolation and characterization. All of the above factors are encountered in the characterization of impurities in rapamycin and hence in rapamycin-based drugs. To overcome these technical challenges, we have used an integrated and systematic approach for the identification of such impurities to compensate for the deficiencies of any particular technology.

To illustrate the utility of this approach, we report the identification of two novel rapamycin derivatives, present in purified rapamycin at low level, using LC-MS/MS, sample isolation by preparative HPLC, followed by detailed structural analysis using multidimensional/ multinuclear NMR spectroscopy in a small volume $1.0 \mathrm{~mm}$ NMR probe. This approach has proven very valuable in the structural identification of a variety of impurities in rapamycin well below the $0.1 \%$ threshold, including several novel fermentation by-products. A similar approach as reported here for rapamycin was successfully applied to characterizing rapamycin-based APIs.

\section{RESULTS AND DISCUSSION}

Figure la shows the structure of rapamycin $\left(\mathrm{R}_{1}=\mathrm{R}_{2}=\mathrm{CH}_{3}\right)$ including the numbering scheme used in this study. A typical feature of the reverse-phase HPLC chromatogram of rapamycin and its derivatives is that the principal peak is always accompanied by a minor, later-eluting peak at an RRT of ca.1.06 (Figure 1b, upper trace). The two peaks are typically observed in approximately a 30:1 ratio and are due to the equilibrating pyran and oxepane hemi-ketal tautomers. ${ }^{20}$ The lower trace in Figure $1 \mathrm{~b}$ shows the same chromatogram at enlarged scale, illustrating a portion of the impurity profile of this material, typical of rapamycin. Here we focus on the arrowed peak at RRT 1.2, which represented a particular challenge for structure determination, requiring the full spectrum of analytical techniques. The peak at RRT 1.2 comprises about $0.1 \%$ of the total sample, while other peaks represent rapamycin-related impurities of different structure. Initial LC-MS results of the RRT 1.2 impurity peak indicated a $m / z$ for the $\mathrm{MNa}^{+}$ ion at $950.6 \mathrm{Da}$, which is $14 \mathrm{amu}$ higher than the $\mathrm{m} / \mathrm{z}$ of sodiated rapamycin $(936.6 \mathrm{Da})$ and consistent with addition of a methylene group. The same mass difference to rapamycin was observed in the mass spectra obtained with several other LC-MS methods (Supplementary Figures S1 and S2) and allowing cross-correlation of retention times of this impurity peak.

Extensive HPLC method development resulted in significantly better resolution between various impurity peaks, albeit at the expense of a significantly increased retention time, highlighting one challenge of characterizing closely related natural product impurity peaks. In the refined HPLC method, the impurity peak was separated into two peaks as shown in the reconstructed single-ion chromatograms (Figure 2b). The $\mathrm{MS}^{2}$ fragmentation spectra of rapamycin and the two impurity peaks are shown in Figures $2 \mathrm{c}-\mathrm{e}$, respectively. At first glance, the fragmentation patterns for the impurities are almost identical, indicating that both peaks might represent tautomers of the same compound. However, closer inspection reveals some differences in fragmentation, which point to structurally distinct compounds. For example, the fragment ion with $\mathrm{m} / \mathrm{z} 642$ was observed in rapamycin and also in impurity 2, while impurity 1 shows a +14 amu difference for this fragment. Similar shifts are found for the fragment at $m / z=320$ (red arrows in Figure 2).

Assignment of the fragmentation pattern was derived as for other rapamycin analogs ${ }^{21-23}$ (see Supplementary Table S3 and Supplementary Figures S3 and S4), and indicates that the mass difference for impurity $\mathbf{1}$ is located in the hemiketal moiety of the macrocycle between $\mathrm{C} 9$ and $\mathrm{C} 13$ (red area in Figure 1a). In contrast, the fragment at $m / z=607$ is conserved between rapamycin and impurity $\mathbf{1}$, but shifted by $+14 \mathrm{amu}$ in impurity 2 . Assignment of fragments (Supplementary Figure S4) reveals that the mass difference for impurity $\mathbf{2}$ is found between C8 and C29 (green area in Figure 1a). The peak at RRT 1.2 in the chromatogram in Figure $1 \mathrm{~b}$ therefore represents two distinct rapamycin impurities, rather than a single peak or indeed equilibrating tautomers. From the regulatory standpoint, while the sum of the two closely eluting compounds exceeds the identification threshold level of $0.1 \%$, individually the compounds are each at or below the reporting threshold $(0.05 \%)$.
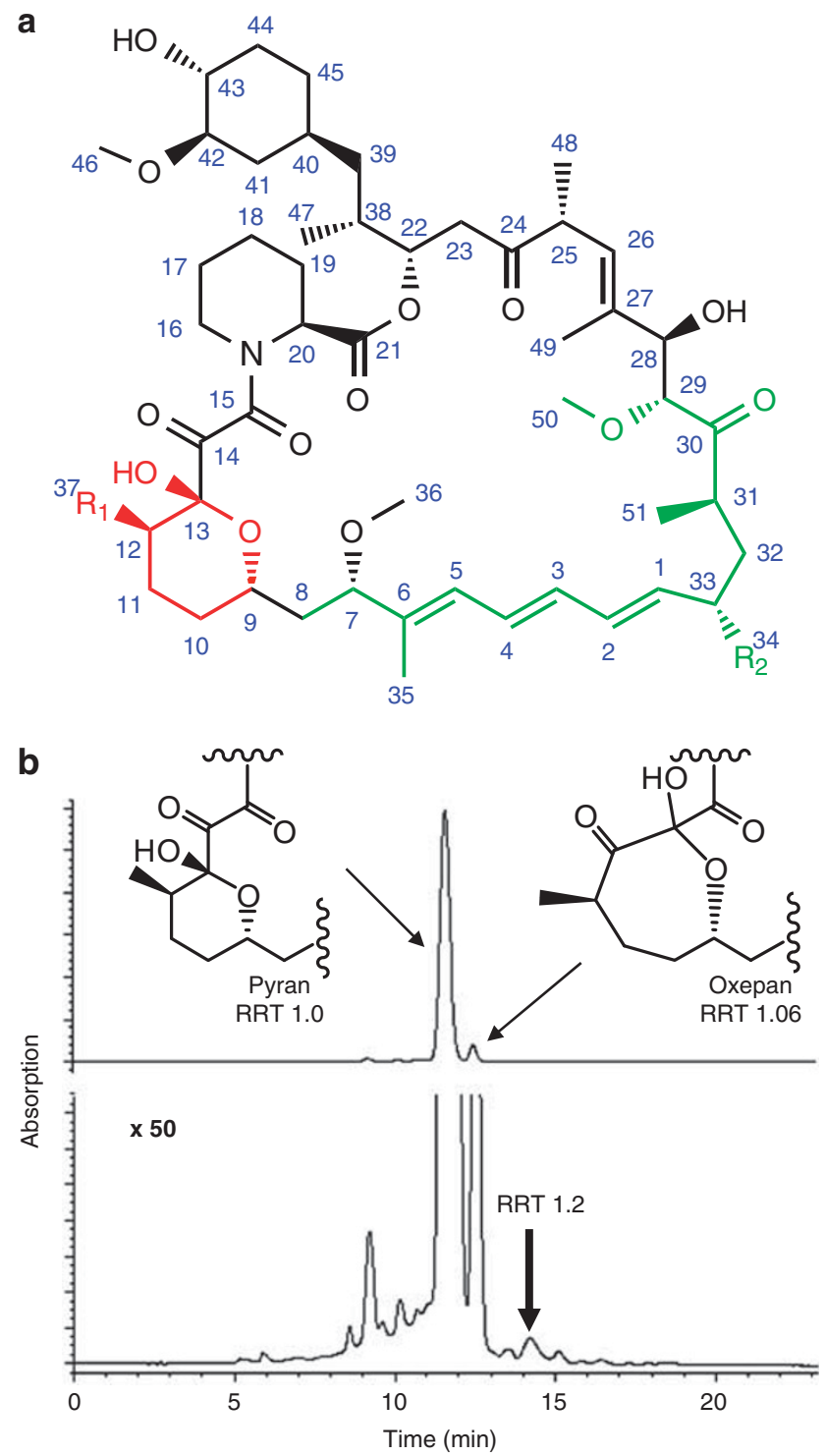

Figure 1 (a) Structure and numbering scheme of rapamycin $\left(R_{1}=R_{2}=C_{3}\right)$, and the API impurities 12-ethyl-rapamycin $\left(\mathrm{R}_{1}=\mathrm{CH}_{2}-\mathrm{CH}_{3}, \mathrm{R}_{2}=\mathrm{CH}_{3}\right)$, and 33-ethyl-rapamycin $\left(\mathrm{R}_{1}=\mathrm{CH}_{3}, \mathrm{R}_{2}=\mathrm{CH}_{2}-\mathrm{CH}_{3}\right)$. The red and green areas mark the location of the +14 amu mass difference from the MS/MS fragmentation pattern for 12-ethyl-rapamycin and 33-ethyl-rapamycin, respectively. (b) Typical HPLC chromatogram for rapamycin API showing two major peaks ascribed to pyran (left) and oxepan (right) tautomers. The lower trace shows the same chromatogram at expanded scale to focus on the impurity profile. The arrow indicates the impurity peak at $\sim 14.1 \mathrm{~min}(R R T=1.2)$ investigated here. 
a

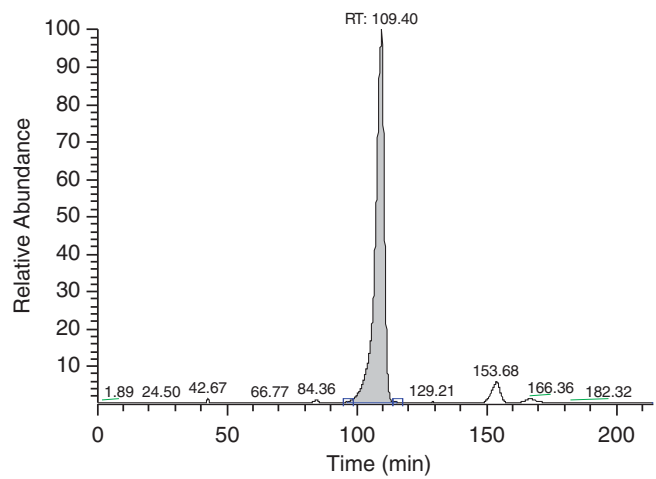

b

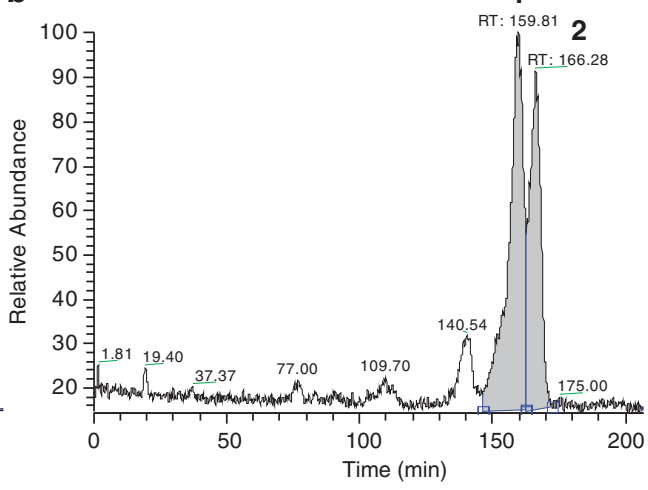

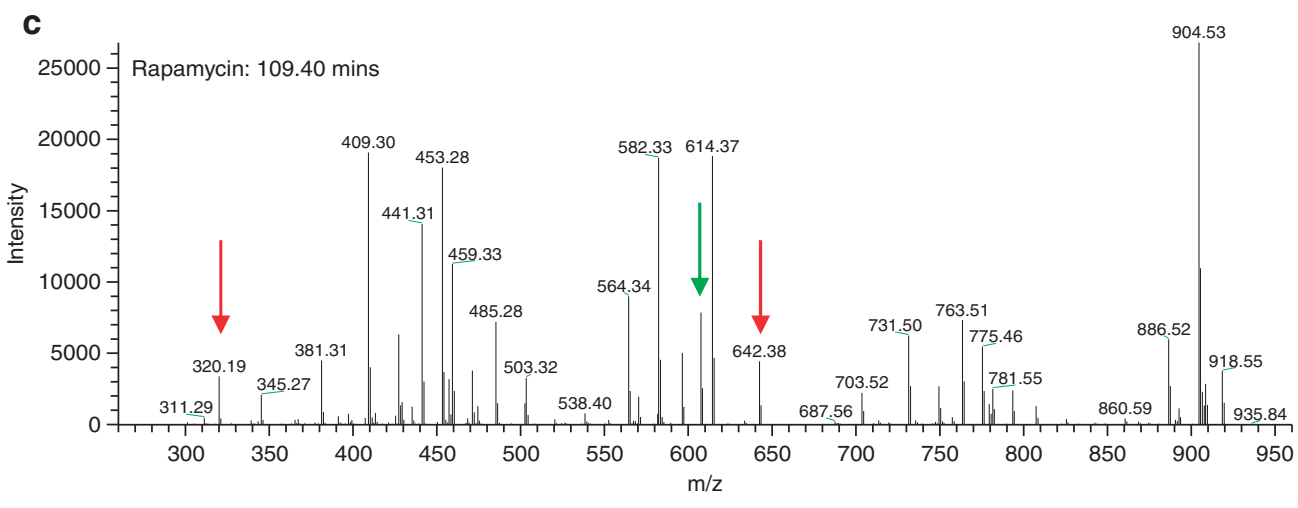

\section{d}

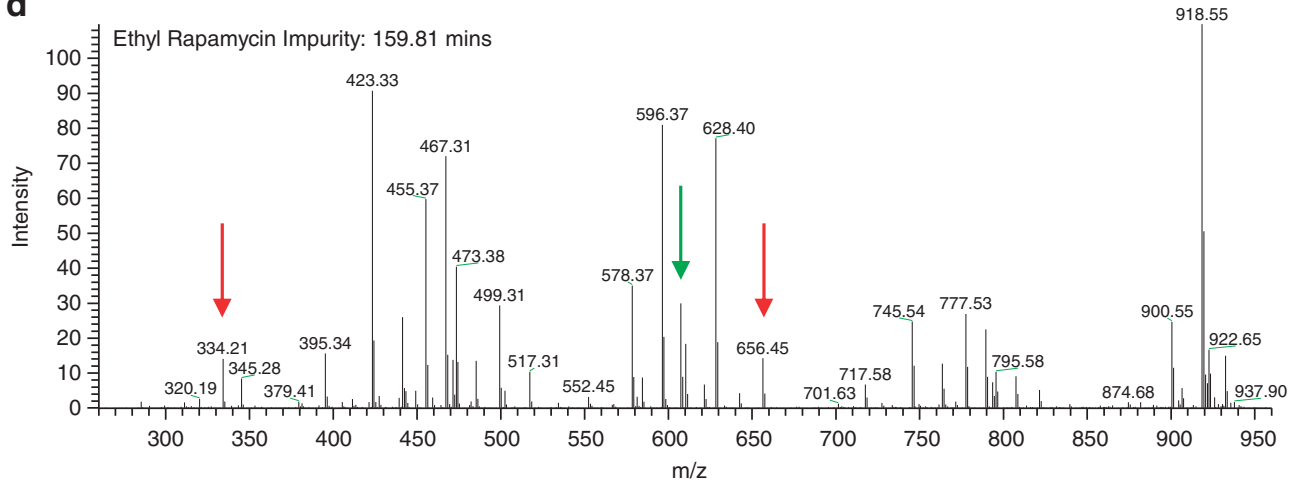

e

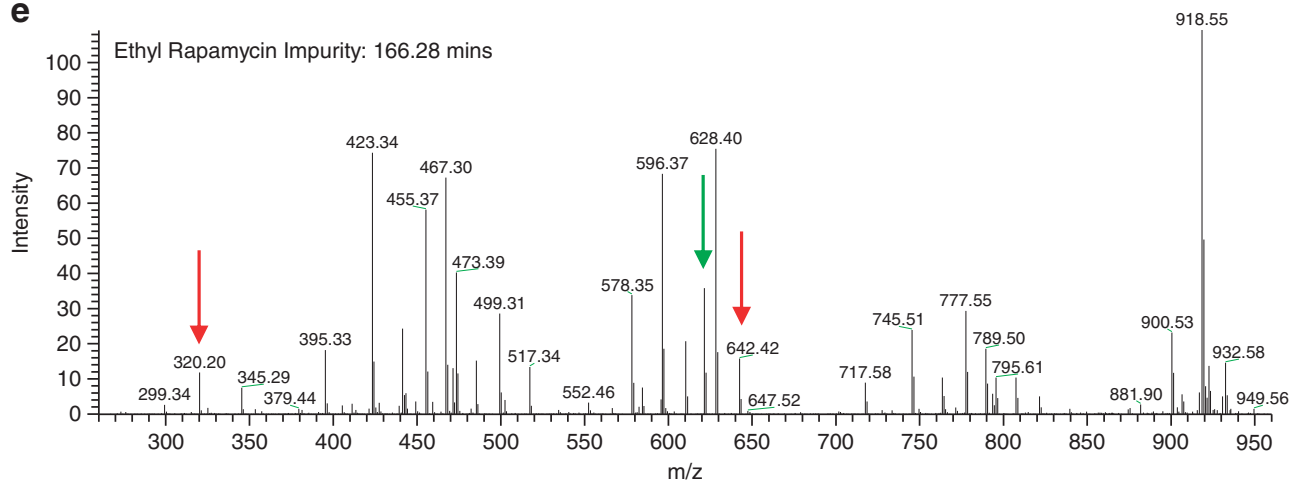

Figure 2 Selected ion MS/MS chromatogram of sodiated rapamycin (a) and all impurities with a +14 amu mass difference (b). MS-MS fragmentation pattern of rapamycin (c) and the impurities at RT=159.8 $\mathrm{min}$ (d) and $166.3 \mathrm{~min}$ (e). The red arrows indicate fragments, which allow locating the mass defect of impurity $\mathbf{1}$ to the hemiketal moiety (red area in Figure 1a). Fragments marked by green arrows locate the mass defect of impurity $\mathbf{2}$ to the green area in Figure 1a. 
On the basis of MS fragmentation alone, however, it was not possible to derive unambiguous structures for these impurities. For impurity 1, an ethyl (instead of methyl) moiety at C12 appears to be the most likely structure, although a methoxy derivative at $\mathrm{C} 13$ is also in agreement with the MS-MS results on 1. A methyl (instead of $\mathrm{H})$ at C10 is also biosynthetically feasible and consistent with the data. Impurity 2 might be an ethyl derivative at either C6, C33 or C51, while an ethoxy derivative at $\mathrm{C} 29$ is also consistent with the MS data. Furthermore, addition of a methylene group in the triene region (C1-C6) is in agreement with the MS data. As the triene region constitutes the major chromophore used for optical quantitation, structural modifications could potentially alter the extinction coefficient and hence lead to miscalculation of the impurity amount. Such structural ambiguities are inadequate for regulatory reporting, necessitating more detailed structure characterization.

The isolation of individual impurities was carried out on two preparative chromatography instruments under isocratic conditions (see Supplementary Information). The RP-HPLC chromatograms of the isolates (Supplementary Figures S5 and S6) are consistent with a RRT of 1.23 and 1.22, respectively. Loop-injection mass spectra for both positive and negative ions (Supplementary Figures S5 and S6) on both samples indicate a molecular weight of 927.6 based on a $\mathrm{m} / \mathrm{z}$ of $945.6\left(\mathrm{M}+\mathrm{NH}_{4}{ }^{+}\right)$in the positive scan and $m / z 926.6\left(\mathrm{M}-\mathrm{H}^{-}\right)$in the negative scan. In agreement with the LC-MS studies discussed above, the molecular weight for both isolates represents a gain of $14 \mathrm{amu}$ from the molecular weight of rapamycin (914.2 Da).

Extensive (1D and 2D) $600 \mathrm{MHz}$ NMR studies on the isolated impurities were conducted comprising gCOSY, gTOCSY, multiplicity edited gHSQC and gHMBC spectra (Supplementary Figures S8-S12). Assignments for all 2D homo- and hetero-nuclear spectra were necessary to provide sufficient experimental evidence for unambiguous identification of rapamycin congeners. Sample capillaries with $1.0 \mathrm{~mm}$ o.d. were used with an inverse gradient NMR microprobe (Bruker Biospin, Karlsruhe, Germany), which provides significantly higher mass sensitivity with respect to the same sample amount compared with $5 \mathrm{~mm}$ conventional probes. This allowed us to acquire all $1 \mathrm{D}$ and $2 \mathrm{D}$ NMR data sets within $\sim 20 \mathrm{~h}$ per sample, even at limited sample amount $(\sim 200 \mathrm{nmol})$. Degradation of the isolated impurities was usually much slower than the time required for NMR data acquisition.

Overlays of the $2 \mathrm{D}{ }^{1} \mathrm{H}_{-}{ }^{13} \mathrm{C}$ HSQC spectra of impurity isolates $\mathbf{1}$ and 2 with rapamycin are shown in Figure 3. Resonance assignments have been obtained as described previously in detail, following the approach for rapamycin analogs reported in the literature. ${ }^{24}$ Rapamycin and both of the isolates exists as a mixture of the trans and cis rotamers about the peptide bond (Supplementary Figure S7). Integration of the peaks assigned to $\mathrm{C} 20$ and $\mathrm{C}^{\prime} 0^{\prime}$ in the HSQC spectra indicates a trans:cis isomer ratio of about $4: 1$ in $\mathrm{CDCl}_{3}$. Assignments were obtained for both rotamers, whenever resolved.

For both impurities, the overall spectra are almost identical for ${ }^{13} \mathrm{C}$ and ${ }^{1} \mathrm{H}$ resonances, and only a few peaks show sizable changes in chemical shifts. For impurity isolate 1 , those include ${ }^{13} \mathrm{C}$ and ${ }^{1} \mathrm{H}$ resonances at positions $\mathrm{C} 32, \mathrm{C} 33$ and the methyl group C34 in rapamycin (blue arrows in Figure 3a). Two new resonances are observed in the aliphatic region and are ascribed to a $\mathrm{CH}_{2}$ group according to their spin multiplicity (Supplementary Figure S11). This indicates only minor changes in the structure of the macrocycle between rapamycin and impurity $\mathbf{1}$, reflecting the difference of a methyl group relative to an ethyl group. The addition of a methoxy $\mathrm{CH}_{3}$ can be excluded from the NMR data. Chemical shift changes and the spin-spin correlations observed in COSY, TOCSY and HMBC
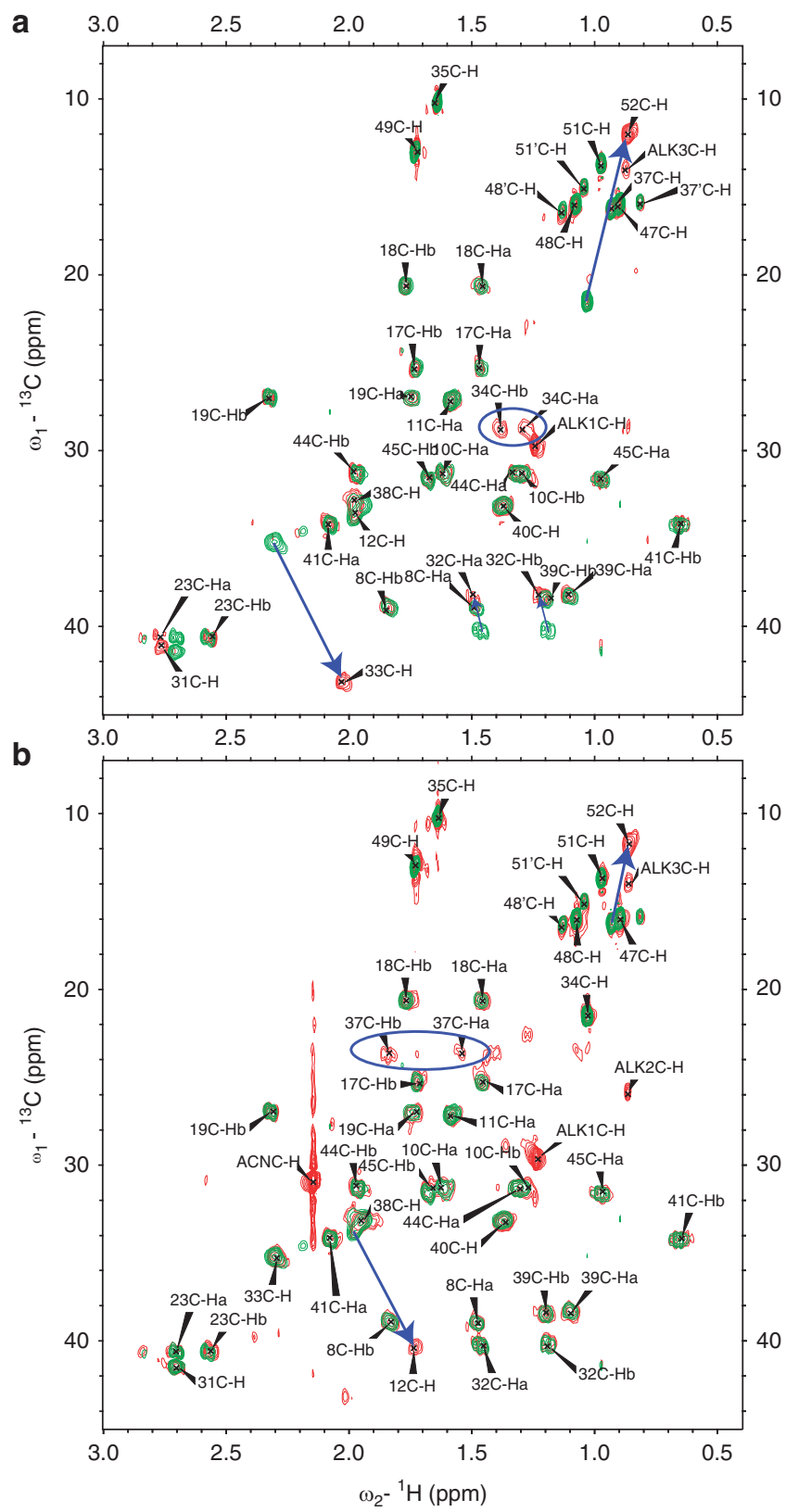

Figure 3 Overlay of the HSQC spectrum of rapamycin (green) and the impurity spectrum (red) for isolate $\mathbf{1}$ (a) and $\mathbf{2}$ (b). The arrows indicate major changes in chemical shifts. Newly appearing $\mathrm{CH}_{2}$ groups are indicated by the blue circles. All assignments correspond to the impurity spectra. Artifacts introduced by the sample isolation process are indicated as ACN (for acetone) and ALK (for alkanes arising from the HPLC column).

spectra, point to a modification at position 34 , and are consistent with the assignment of this isolate as 33-ethyl-rapamycin (Figure 1, $\left.\mathrm{R}_{1}=\mathrm{CH}_{3}, \mathrm{R}_{2}=\mathrm{CH}_{2}-\mathrm{CH}_{3}\right)$. On the other hand, in Figure $3 \mathrm{~b}$, shifted resonances for impurity isolate 2 include ${ }^{13} \mathrm{C}$ and ${ }^{1} \mathrm{H}$ resonances at positions $\mathrm{C} 12, \mathrm{C} 11$ and the methyl group $\mathrm{C} 37$ in rapamycin. Two new $\mathrm{CH}_{2}$ resonances are observed in the aliphatic region. These changes, again reflect the difference of a methyl group relative to an ethyl group, and exclude the addition of a methoxy group. Chemical shift changes and spin-spin correlations for isolate 2 (Supplementary Figures S13-S17) point to a modification at position 12 , and are 
consistent with the assignment of this species as 12-ethyl-rapamycin (Figure 1, $\mathrm{R}_{1}=\mathrm{CH}_{2}-\mathrm{CH}_{3}, \mathrm{R}_{2}=\mathrm{CH}_{3}$ ). Chemical shifts for both impurities are reported in the Supplementary Tables S4 and S5.

Closer comparison reveals that isolate $\mathbf{2}$ is a mixture of compounds 12-ethyl-rapamycin and 33-ethyl-rapamycin, with the former being the major component (ca. 70\%). Similarly, the LC-MS/MS spectra for isolate $\mathbf{2}$ indicated mixtures between the two ethyl-rapamycin derivatives, with 12-ethyl-rapamycin being the dominant component. This observation is explained by the overlap in the HPLC chromatogram between the oxepan tautomer of 33-ethyl-rapamycin and the pyran tautomer of 12-ethyl-rapamycin. Upon isolation, the oxepan tautomer of 33-ethyl-rapamycin re-equilibrates into its pyran tautomer, leading to the observed mixture of species in isolate 2 .

Ethyl-rapamycin congeners were present in several lots of rapamycin from different vendors, although at varying concentrations depending on the purification procedures used in API production. As an illustration, Supplementary Figure S18 shows selected ion MS/MS chromatograms of impurities with a +14 -amu mass defect for several lots of CCSB rapamycin. The shaded areas around RRT 1.2 are representing the ethyl-rapamycin species discussed herein.

\section{EXPERIMENTAL PROCEDURE}

\section{Source material}

As rapamycin is an approved drug, API is commercially available from various suppliers. The material used in this study was manufactured under GMP regulations by Chunghwa Chemical Synthesis \& Biotech, (CCSB, Taipei, Taiwan, Lot 96186001), using an optimized strain derived from the original Streptomyces rapamycinicus ATC strain.

\section{Analytical HPLC}

HPLC analyses of rapamycin, impurity pools and isolated individual impurities were carried out on Agilent 1100 and HP1050 systems running ChemStation Software A.10.02 (Agilent, Santa Clara, CA, USA). Each system was equipped with a quarternary pump degasser, an autosampler and a diode array detector. Injection volume was limited to $5 \mu \mathrm{l}$ in 1:1 methanol: buffer to suit the nature and quantity of the isolate. In many cases, isolates were injected directly as solutions in unbuffered preparative chromatography eluent. The relative retention times (RRT) of isolates were established or confirmed by co-injection with an approximately equimolar solution of rapamycin. Detailed conditions are described in Supplementary Tables S1A and S1B.

\section{Isolation of individual impurities by preparative HPLC}

A measure of $4.5 \mathrm{~g}$ of rapamycin were dissolved at $0.1 \mathrm{~g} \mathrm{ml}^{-1}$ in 1:1 acetonitrile:water and injected on prep method 1 (Supplementary Information), in 30 $150 \mathrm{mg}$ aliquots. Eluent containing late-eluting impurities (RRT $>1$ ) was pooled and concentrated to yield a 50-mg pool of impurities. The impurity pools were subsequently fractionated by iterative prep-HPLC runs, using both prep method 1 and prep method 2 (Supplementary Table S2), with varying mobile phase systems, to arrive at impurity isolates of sufficient purity for characterization. For the impurities at RRT 1.2, an amount of 200-400 $\mu \mathrm{g}$ per sample, suitable for NMR structure identification, was isolated from $\sim 4.5 \mathrm{~g}$ of starting material within a few days.

\section{LC-MS/MS}

LC-MS/MS was conducted on a Finnigan Surveyor HPLC system consisting of a MS-pump plus, an autosampler plus and a PDA plus detector (Thermo Electron, San Jose, CA, USA). The mobile phase consisted of two solvents A $(0.1 \%$ formic acid in water) and B (100\% Methanol). Sample components were separated on a Waters (Milford, MA, USA) YMC-ODS AQ column $(4.6 \times 250 \mathrm{~mm}, 5 \mu \mathrm{m})$ using an isocratic method at $61 \% \mathrm{~B}$. The mobile phase flow rate was $1.0 \mathrm{ml}$ per min, of which $0.5 \mathrm{ml}$ per min was directed to the MS using a splitter. The working stock solution $\left(30 \mu \mathrm{l}\right.$ of a $50 \mu \mathrm{g} \mathrm{ml}^{-1}$ rapamycin solution in 1/1 methanol/water) was injected onto the Thermo Finnigan LC/ LTQ linear ion trap MS. The MS was operated in positive-ion mode under electrospray ionization conditions with a capillary temperature of $270{ }^{\circ} \mathrm{C}$, spray voltage of $4 \mathrm{kV}$ and collision energy of $35 \%$. Full-scan $\mathrm{MS}^{1}$ and $\mathrm{MS}^{2}$ spectra of the sodium adduct of the major molecular ion were collected.

\section{NMR spectroscopy}

Samples of the isolated impurities were prepared by dissolving ca. $200-400 \mu \mathrm{g}$ of crude isolate in $10 \mu \mathrm{l}$ of deuterated chloroform $\left(\mathrm{CDCl}_{3}\right.$, Cambridge Isotope Labs, Andover, MA, USA). Before sample preparation, the $\mathrm{CDCl}_{3}$ was filtered through basic alumina to remove acidic impurities. NMR spectra were acquired at a temperature of $300 \mathrm{~K}$ on a Bruker Avance DRX-600 NMR spectrometer equipped with a 1 -mm three-channel $\left({ }^{1} \mathrm{H},{ }^{13} \mathrm{C},{ }^{15} \mathrm{~N}\right) z$-gradient microprobe. All ${ }^{1} \mathrm{H}$ chemical shifts were referenced to the residual $\mathrm{CHCl}_{3}$ solvent peak at 7.26 p.p.m. Assignment of proton signals was obtained from $1 \mathrm{D}{ }^{1} \mathrm{H}$ spectra, and 2D gradient enhanced DQ-COSY and TOCSY spectra with $60 \mathrm{~ms}$ DIPSI-2 spin lock pulse. ${ }^{13} \mathrm{C}$ chemical shifts were obtained from gradient-enhanced HSQC spectra using Garp ${ }^{13} \mathrm{C}$ decoupling during detection. ${ }^{13} \mathrm{C}$ shifts for quaternary carbon atoms and carbonyls were derived from gradient enhanced HMBC spectra. All data were processed using the Bruker Topspin 1.3 software. Peak assignments were made using Sparky V3.1. Chemical shifts represent the average between the corresponding peaks in all 2D NMR spectra.

Experimental details on sample isolation as well as MS/MS fragmentation and NMR data on the isolated impurities are available as Supporting Information.

\section{CONCLUSION}

Rapamycin and its derivatives are complex natural products and, in consequence, the identification of low-level structurally related impurities present in purified samples provides a significant analytical challenge. Combining LC-MS/MS sample isolation by prep-HPLC and multidimensional NMR spectroscopy proved to be powerful and reliable workflow for impurity structure elucidation. The superior sensitivity of LC-MS can be used to analyze various batches of API by monitoring changes in molecular weight of drug-related impurities without the need for isolation of individual impurities. MS fragmentation data are then used to localize the mass difference to a certain part of the molecule. NMR spectroscopy can provide more detailed structural characterization. However, NMR usually requires significant sample amounts due to its relatively low sensitivity. This problem has been alleviated in recent years by the invention of small volume probes and cryo-probes with significantly increased sensitivity. ${ }^{25,26}$ Here, the improved mass sensitivity afforded by a small-volume probe NMR allowed a reduction in the scale of chromatography required to isolate impurities, leading to a faster and less contaminant-prone isolation procedure. With such an approach, NMR was successfully used to pinpoint structural changes at atomic level, hence allowing unambiguous structure identification even for structural isomers with identical molecular weight.

Most of the derivatives isolated from purified API were previously known, such as demethyl- 27 or demethoxy-, ${ }^{28}$ seco-$^{23}$ and prolylrapamycin. ${ }^{29,30}$ However, several novel rapamycin congeners were found, two of which are reported here, as an illustration of the utility of the approach. The ethyl-rapamycin congeners at RRT 1.2 were present in all API lots from CCSB investigated so far, although at amounts well below the identification threshold of $0.10 \%$. In general, the level of fermentation by-products depends not only on the specific strain used in fermentation, but also on timing and amount of precursor feeding, as well as the purification procedures used for API production. A more comprehensive analysis of the impurity profiles of rapamycin from various vendors is beyond the scope of this study and will be given elsewhere.

Both ethyl-rapamycin derivatives discovered here can be easily rationalized as fermentation by-products. According to the biosynthetic pathway of rapamycin, ${ }^{31-33}$ the methyl groups are the result of acyltransferase incorporating a methylmalonyl-CoA extender unit. 
Consequently, ethyl-substituted rapamycin derivatives are the result of incorporation of an ethylmalonyl-CoA extender. Novel rapamycin derivatives have been generated previously using either chemical modification, ${ }^{34-36}$ genetic engineering ${ }^{33,37}$ or were derived by precursor-directed biosynthesis. $22,30,33,38-40$ These biosynthetic derivatives usually comprise changes within the pipecolic-acid moiety, the cyclohexene moiety or involve the methoxy and carboxyl groups of the macrocycle. However, changes in the carbon skeleton of the polyketide have not been reported so far. Hence, using the appropriate biosynthetic precursors may broaden the scope of engineering of new rapamycin derivatives. As the titer of many derivatives can be altered; for example, by feeding biosynthetic precursors as nutrients during fermentation, ${ }^{30,38,39}$ full structural characterization of fermentation by-products is a requirement to minimize unwanted congeners. With both ethyl-rapamycin derivatives present below the reportable level, further in vitro potency or in vivo toxicological evaluation was neither necessary for regulatory purposes nor feasible considering the submilligram amounts available by isolation from purified API. However, using the appropriate biosynthetic precursors, the amount of ethylrapamycin congeners may be significantly increased, thus facilitating isolation at larger scale; for example, directly from fermentation broth to support various biophysical, in vitro and in vivo studies, which require notably more material than the structure elucidation described herein. To extend its utility, structure determination of low-level impurities by the methodology described above can also be applied to other rapamycin analog APIs, as well as to the identification of drug metabolites present at low levels, and to the elucidation of the structure of natural product precursors and degradants with potentially novel bioactivity.

\section{ACKNOWLEDGEMENTS}

We thank Barry Davis, Karin Russian and Joseph Snodgrass for assisting in the acquisition and analysis of LC-MS/MS data on some of the isolated impurities and helpful discussions.

1 Sehgal, S., Baker, H. \& Vezina, C. Rapamycin (AY-22,989), a new antifungal antibiotic. II. Fermentation, isolation and characterization. J. Antibiot. 28, 727-732 (1975).

2 Kumar, Y. \& Goodfellow, M. Five new members of the Streptomyces violaceusniger $16 \mathrm{~S}$ rRNA gene clade: Streptomyces castelarensis sp. nov., comb. nov., Streptomyces himastatinicus sp. nov., Streptomyces mordarskii sp. nov., Streptomyces rapamycinicus sp. nov. and Streptomyces ruanii sp. nov. Int. J. Syst. Evol. Microbiol. 58, 1369-1378 (2008).

3 Calne, R. Y. et al. Rapamycin for immunosuppression in organ allografting. Lancet 2, 227 (1989).

4 Watson, C. J. E. et al. Sirolimus: a potent new immunosuppressant for liver transplantation. Transplantation 67, 505-509 (1999).

5 Marx, S. O. \& Marks, A. R. Bench to bedside: the development of rapamycin and its application to stent restenosis. Circulation 104, 852-855 (2001).

6 Woods, T. C. \& Marks, A. R. Drug-eluting stents. Medicine 55, 169-178 (2004).

7 Luscher, T. F. et al. Drug-eluting stent and coronary thrombosis: biological mechanisms and clinical implications. Circulation 115, 1051-1058 (2007).

8 Neuhaus, P., Klupp, J. \& Langrehr, J. M. mTOR inhibitors: an overview. Liver Transplantation 7, 473-484 (2001).

9 Sabatini, D. M. mTOR and cancer: insights into a complex relationship. Nat. Rev. Cancer 6, 729-734 (2006).
10 Faivre, S., Kroemer, G. \& Raymond, E. Current development of mTOR inhibitors as anticancer agents. Nat. Rev. Drug Discov. 5, 671-688 (2006).

11 Harrison, D. et al. Rapamycin fed late in life extends lifespan in genetically heterogeneous mice. Nature 460, 392-395 (2009).

12 Blay, J. Y. Updating progress in sarcoma therapy with mTOR inhibitors. Annals Oncol. 22, 280-287 (2011)

13 Gu, J., Ruppen, M. E. \& Cai, P. Lipase-catalyzed regioselective esterification of rapamycin: synthesis of temsirolimus (CCI-779). Org. Lett. 7, 3945-3948 (2005).

14 Ahuja, S. \& Alsante, K. M. Handbook of Isolation and Characterization of Impurities in Pharmaceuticals (Academic Press, San Diego, CA, USA, 2003).

15 Branch, S. K. Guidelines from the international conference on harmonisation (ICH). J. Pharm. Biomed. Anal. 38, 798-805 (2005).

16 Holzgrabe, U., Wawer, I. \& Diehl, B. NMR Spectroscopy in Drug Development and Analysis (Wiley Online Library, 1999).

17 Alsante, K. M. et al. Pharmaceutical impurity identification: a case study using a multidisciplinary approach. J. Pharm. Sci. 93, 2296-2309 (2004).

18 Smith, R. J. \& Webb, M. L. Analysis of Drug Impurities (Wiley-Blackwell, Oxford, UK, 2007).

19 Marín, A., Espada, A., Vidal, P. \& Barbas, C. Major degradation product identified in several pharmaceutical formulations against the common cold. Anal. Chem. 77, 471-477 (2005).

20 Hughes, P., Musser, J., Conklin, M. \& Russo, R. The isolation, synthesis and characterization of an isomeric form of rapamycin. Tetrahedron Lett. 33, 4739-4742 (1992).

21 Hallensleben, K., Raida, M. \& Habermehl, G. Identification of a new metabolite of macrolide immunosuppressant, like rapamycin and SDZ RAD, using high performance liquid chromatography and electrospray tandem mass spectrometry. J. Am. Soc. Mass Spec. 11, 516-525 (2000).

22 Gregory, M. A. et al. Rapamycin biosynthesis: elucidation of gene product function. Org. Biomol. Chem. 4, 3565-3568 (2006).

23 Cai, P., Tsao, R. \& Ruppen, M. E. In vitro metabolic study of temsirolimus: preparation, isolation, and identification of the metabolites. Drug Metab. Dispos. 35, 1554-1563 (2007).

24 Kessler, H., Haessner, R. \& Schüler, W. Structure of rapamycin: an NMR and molecular dynamics investigation. Helv. Chim. Acta. 76, 117-130 (1993).

25 Hopson, R. E. \& Peti, W. Microcoil NMR spectroscopy: a novel tool for biological high throughput NMR spectroscopy. Meth. Mol. Biol. 426, 447-458 (2008).

26 Martin, G. E. \& Webb, G. A. Small-Volume and High-Sensitivity NMR Probes. Annual Reports on NMR Spectroscopy. pp 1 (Academic Press, San Diego, CA, USA, 2005).

27 Box, S. J. et al. 27-0-demethylrapamycin, an immunosuppressant compound produced by a new strain of Streptomyces hygroscopicus. J. Antibiot. 48, 1347 (1995).

28 Findlay, J. A., Liu, J. S., Burnell, D. J. \& Nakashima, T. T. The structure of demethoxyrapamycin. Can. J. Chem. 60, 2046-2047 (1982).

29 Wong, G. K., Griffith, S., Kojima, I. \& Demain, A. L. Antifungal activities of rapamycin and its derivatives, prolylrapamycin, 32-desmethylrapamycin, and 32-desmethoxyrapamycin. J. Antibiot. 51, 487 (1998).

30 Ritacco, F. V. et al. Production of novel rapamycin analogs by precursor-directed biosynthesis. App. Environ. Microbiol. 71, 1971-1976 (2005).

31 Schwecke, T. et al. The biosynthetic gene cluster for the polyketide immunosuppressant rapamycin. Proc. Natl Acad. Sci. USA. 92, 7839-7843 (1995).

32 Staunton, J. \& Wilkinson, B. Biosynthesis of erythromycin and rapamycin. Chem. Rev. 97, 2611-2630 (1997).

33 Park, S. R., Yoo, Y. J., Ban, Y.- H. \& Yoon, Y. J. Biosynthesis of rapamycin and its regulation: past achievements and recent progress. J Antibiot. 63, 434-441 (2010).

34 Yang, W. et al. Selective epimerization of rapamycin via a retroaldol/aldol mechanism mediated by titanium tetraisopropoxide. Org. Lett. 1, 2033-2035 (1999).

35 Luengo, J. I., Konialian, A. L. \& Holt, D. A. Studies on the chemistry of rapamycin: novel transformations under Lewis-acid catalysis. Tetrahedron Lett. 34, 991-994 (1993).

36 Luengo, J. I. et al. Structure-activity studies of rapamycin analogs: evidence that the C-7 methoxy group is part of the effector domain and positioned at the FKBP12-FRAP interface. Chem. Biol. 2, 471-481 (1995).

37 Chung, L., Liu, L., Patel, S., Carney, J. R. \& Reeves, C. D. Deletion of rapQONML from the rapamycin gene cluster of Streptomyces hygroscopicus gives production of the 16-O-desmethyl-27-desmethoxy analog. J. Antibiot. 54, 250 (2001).

38 Lowden, P. A., Bohm, G. A., Metcalfe, S., Staunton, J. \& Leadlay, P. F. New rapamycin derivatives by precursor-directed biosynthesis. Chem. BioChem. 5, 535-538 (2004).

39 Goss, R. J. M. et al. An expeditious route to fluorinated rapamycin analogues by utilising mutasynthesis. Chem. BioChem. 11, 698-702 (2010).

40 Nishida, H. et al. Generation of novel rapamycin structures by microbial manipulations. J. Antibiot. 48, 657-666 (1995).

Supplementary Information accompanies the paper on The Journal of Antibiotics website (http://www.nature.com/ja) 